\title{
Endophilin A2 regulates B cell protein trafficking and humoral responses
}

\author{
Dessislava Malinova ${ }^{1,2,3, *}$, Laabiah Wasim ${ }^{1}$, Niklas Engels ${ }^{4}$, Pavel Tolar ${ }^{1,2, *}$ \\ ${ }^{1}$ Immune Receptor Activation Laboratory, The Francis Crick Institute, London, NW1 1AT, \\ United Kingdom. \\ ${ }^{2}$ Division of Immunology \& Inflammation, Department of Medicine, Imperial College \\ London, London, SW7 2A2, United Kingdom. \\ ${ }^{3}$ Wellcome-Wolfson Institute for Experimental Medicine, Queen's University Belfast, \\ Belfast, BT9 7BL, United Kingdom. \\ ${ }^{4}$ Institute of Cellular \& Molecular Immunology, University Medical Center Göttingen, \\ Göttingen, Germany \\ *Corresponding authors; Lead contact: pavel.tolar@crick.ac.uk
}

\section{HIGHLIGHTS}

- Genome-wide CRISPR screens comprehensively identify genes regulating antigen uptake in B cells

- B cell receptor-mediated antigen internalization is mediated by both epsin1- dependent clathrincoated pits and a novel fast endophilin A2-mediated endocytosis.

- Endophilin A2 is required for peripheral B cell development, antigen-specific germinal center responses and high-affinity $\operatorname{IgG}$ production. 
- Endophilin A2 is broadly essential for B cell intracellular trafficking pathways providing metabolic support of germinal center B cell proliferation, in part through regulation of iron uptake.

\begin{abstract}
Antigen-specific B cell responses require endosomal trafficking to regulate antigen uptake and presentation to helper T cells, and to control expression and signaling of immune receptors. However, the molecular composition of B cell endosomal trafficking pathways and their specific roles in B cell responses have not been systematically investigated. Here we report high-throughput identification of genes regulating $\mathrm{B}$ cell receptor (BCR)-mediated antigen internalization using genome-wide functional screens. We show that antigen internalization depends both on clathrin-coated pits and on clathrinindependent endocytosis mediated by endophilin A2. Although endophilin A2 is dispensable for presentation of the endocytosed antigen, it is required for metabolic support of germinal center (GC) B cell proliferation, in part through regulation of iron uptake. Consequently, endophilin A2 deficient mice show selective defects in GC B cell responses and production of high-affinity IgG. The requirement for endophilin A2 highlights a unique importance of clathrin-independent intracellular trafficking in GC B cell clonal expansion and antibody responses.
\end{abstract}




\section{INTRODUCTION}

Robust antibody responses are crucially dependent on the B cells' ability to internalize, process and present foreign antigens. Antigens are recognized by the B cell receptor (BCR), and their binding triggers rapid BCR endocytosis and trafficking into lysosomal antigen-processing compartments, delivering antigenic peptides for loading onto MHC class II molecules (MHCII) ${ }^{1,2}$. Display of peptideloaded MHCII on B cell surfaces solicits interaction with helper T cells, which, together with antigeninduced BCR signaling, stimulates metabolic and transcriptional B cell activation that culminates in formation of germinal centers (GCs) and production of high affinity IgG antibodies. By controlling the number and repertoire of peptide-loaded MHCII molecules available for recognition by cognate T cells, BCR-mediated antigen internalization thus critically determines the outcome of B cell responses. Furthermore, endocytic trafficking of the BCR modulates BCR signaling strength and duration, and performs homeostatic functions which need to be regulated alongside immune-specific functions in the rapidly proliferating activated $\mathrm{B}$ cells ${ }^{3}$. Consequently, $\mathrm{B}$ cell responses are sensitive to mutations or inhibitors impairing the endolysosomal system $^{4-7}$. However, the molecular composition of B cell endocytic and intracellular trafficking pathways remains poorly understood.

The best characterized mechanism of BCR internalization from the cell surface is clathrin-mediated endocytosis $(\mathrm{CME})^{8-10}$. Clathrin is recruited to the BCR through its adaptor AP-2, which binds to the intracellular tyrosine-based activation motifs (ITAMs) in the CD79A and CD79B subunits ${ }^{10,11}$. In addition, antigen binding to the BCR regulates CME by Lyn-mediated phosphorylation of the clathrin heavy chain ${ }^{10,12}$, promoting its binding to clathrin light chain and coupling of clathrin-coated pits (CCPs) to the actin cytoskeleton ${ }^{13}$. Activated BCRs also associate with E3-ubiquitin ligases c-Cbl and Cbl-b, resulting in ubiquitination of the BCR CD79A and CD79B subunits, which likely plays a role in both BCR-antigen internalization and downstream intracellular trafficking ${ }^{14}$. Recent proteomic studies have further substantiated extensive links between BCR signaling and CCP components ${ }^{15}$.

However, CME is unlikely to be exclusively responsible for antigen-induced BCR internalization. For example, genetic depletion of clathrin only partly reduced BCR endocytosis, revealing another, 
cholesterol and actin-dependent pathway ${ }^{12}$. Furthermore, within seconds of antigen binding, the ITAMs of the BCR are tyrosine-phosphorylated, which abrogates AP-2 binding, predicting that BCR signaling and internalization are mutually exclusive for individual receptors ${ }^{11,16}$. This is paradoxical, as antigen binding dramatically increases, rather than reduces, the speed of BCR endocytosis. Thus, the BCR may use several alternative mechanisms of endocytosis.

Recently, our understanding of endocytic pathways has been broadened by characterization of a clathrin-independent endocytic route termed fast endophilin-mediated endocytosis (FEME). FEME is mediated by the endophilin A family of membrane trafficking proteins, which sense and induce membrane curvature through their BAR domains ${ }^{17,18}$. Endophilins were originally described as components of intracellular clathrin-coated vesicles in neurons ${ }^{19}$. However, recent data from nonneuronal cells identify FEME as a clathrin-independent ligand-triggered endocytic mechanism for a range of cell surface receptors, including growth factor receptors and G-protein coupled receptors, and for bacterial toxins ${ }^{20-22}$. FEME shares many requirements with CME, such as the need for actin cytoskeleton and dynamin2, making it difficult to distinguish from CME using traditional pharmacological treatments ${ }^{21,23}$. However, FEME requires ligand-induced assembly of receptor signaling complexes in order to recruit endophilin A proteins to the plasma membrane via their SH3 domains ${ }^{21}$.

In addition to FEME, endophilins have been implicated in a number of membrane remodelling processes including formation of podosomes ${ }^{24}$, autophagy ${ }^{25}$ and cellular polarity ${ }^{26}$. Through regulation of vesicle trafficking, endophilins are also essential for maintenance of neuronal health ${ }^{19,25,27}$ and have been strongly linked to neurodegeneration in Parkinson's disease ${ }^{25,28}$. However, the molecular mechanisms of endophilin function in neurons and other cell types remain to be debated.

Here, using unbiased genetic screening in B cells we characterize the molecular components of antigen endocytosis and uncover a novel role for endophilin A2, the only member of the endophilin A family expressed in haematopoietic cells, in clathrin-independent antigen-stimulated internalization of the BCR. We also show that in addition to regulating BCR endocytosis, endophilin A2 is essential in activated B cells to maintain mitochondrial respiration via regulation of iron uptake. Consequently, 
mice deficient in endophilin A2 show impaired expansion of GC B cells and poor production of highaffinity IgG antibodies. These results point to a selective importance of endophilin A2-mediated endocytic trafficking in GC B cell responses.

\section{RESULTS}

\section{Improved CRISPR-based screening allows annotation of the antigen uptake pathway}

To comprehensively identify genes regulating BCR-mediated antigen uptake in B cells using CRISPR screening, we generated a Ramos cell line stably expressing the $S$ pyogenes Cas9 nuclease. The human Burkitt's lymphoma line has intact tonic and ligand-induced BCR signaling and efficient endocytosis of surrogate antigen, anti-IgM $F\left(a^{\prime}\right)_{2}$ fragments. We used second generation lentiviral vectors to transduce two pooled genome-wide CRISPR/Cas9 libraries - $\mathrm{GeCKO}^{25}$ and Brunello ${ }^{29}$. After drug selection for sgRNA expression, we screened for genetic disruptions affecting antigen endocytosis using a soluble fluorescent surrogate antigen internalization assay and flow cell sorting (Fig 1A). We extracted genomic DNA from sorted and total populations, amplified the integrated viral genomes and analyzed the abundance of sgRNAs by next-generation sequencing. Depletion or enrichment of sgRNAs in sorted populations expressed as a $\log 2$ ratio of sgRNA abundance, called here internalization score, allows us to infer the contribution of targeted genes to antigen internalization.

To asses library targeting efficiency, we have also determined a CRISPR survival score as the $\log 2$ ratio of sgRNA abundance pre- and post- drug selection. We found that survival scores of previously described essential genes ${ }^{30}$ were significantly lower than survival scores of non-targeting controls (Fig S1A,C). However, the reduction of essential-gene survival scores was greater with Brunnello library. Thus large-scale gene disruption is functional with both libraries, but is more efficient with the Brunello library in line with its optimized on-target efficiency ${ }^{29}$.

Analysis of gene internalization scores from GeCKO library showed that among the top hits with a positive role in antigen internalization included components of CCPs (EPN1, PICALM), intracellular trafficking (RAB7A, VPS16), regulators of the actin cytoskeleton (ACTR2, CCZ1B), exocyst components (EXOC7) and BCR signaling components (GRB2, LYN, CBL), thus establishing that the 
screen is detecting realistic effects (Fig1B). Surprisingly, the screen also identified Endophilin A2 (encoded by $S H 3 G L 1$ ), a critical component of FEME. We validated the key endocytic genes, including SH3GL1, using individual sgRNAs, as elaborated below. However, the statistical significance of the hits from the GeCKO library screen was generally low (Supplementary Table 1) and the validation rate of other candidates was poor (not shown). This was likely due to the low efficiency of gene targeting, supported by an inverse correlation between the predicted sgRNA on-target efficiency and survival scores calculated for the known essential genes (Fig S1B).

The more recent Brunello library showed higher statistical significance for top screen hits (Fig 1C, Supplementary Table 2). The hits included regulators of endocytosis (EPN1, EPS15, PICALM), as well as genes predicted to be involved in a wide range of cellular functions. To validate gene candidates in a high-throughput manner, we re-screened using a custom CRISPR sgRNA minilibrary containing 3 sgRNAs per gene for the top 213 positive or negative regulators, which were also highly expressed in Ramos cells (Supplementary Table 3). The custom minilibrary also contained 15 essential genes as positive controls, and 48 non-targeting sgRNA controls. Analysis of essential gene targeting again showed efficient genetic disruption by the custom library (Fig S1E). The internalization re-screen validated the function of 72 genes with strong statistical significance $(q<0.05)$ (Fig 1D, Supplementary Table 3), including 58 positive regulators (score $<-0.4$ ) and 14 negative regulators (score $>0.35$ ), covering a broad range of cellular processes such as endocytosis, endosomal and lysosomal trafficking, ubiquitination, actin cytoskeleton, Golgi and ER biology, and transcriptional regulation (Fig 1E).

Together, these experiments represent the first comprehensive analysis of the genetic make-up of the BCR internalization pathway and strongly implicate specific endocytic genes as well as downstream feedback from a large and complex regulatory network.

\section{Endophilin A2 is involved in antigen-dependent BCR internalization}

To understand the role of candidate genes on antigen uptake mechanistically, we focused on genes predicted to directly regulate endocytosis. Overall, the screen results confirmed the role of CCPs, suggesting that BCR-mediated antigen endocytosis is particularly dependent on a subset of CCP 
components, such as epsin 1 (EPN1), PICALM and HIP1. However, the data also implicated endophilin A2, suggesting a potential role of clathrin-independent endocytosis. We validated the involvement of the key genes by individual CRISPR-mediated knockouts in Ramos-Cas9 cells (Fig 2A, B) and in primary B cells (Fig 2C). As endophilin A2 has not been studied in B cells before, we focused on its role in subsequent studies. In both Ramos cells and primary mouse B cells, targeting of endophilin A2 reduced soluble antigen uptake to an extent comparable with targeting CME (Fig 2B, C). SH3GL1 loss also strongly impaired internalization of antigen from immune synapses with membrane substrates (Fig 2D). However, endophilin A2 was important only for internalization of an activating anti-IgM F(ab')2 fragment, but not for a non-stimulatory anti-IgM Fab fragment, whereas CME regulated internalization of both (Fig 2E). Thus, endophilin A2 in an important novel player selectively involved in BCR internalization upon antigen ligation.

To determine if endophilin A2 regulates BCR endocytosis directly, we overexpressed endophilin A2GFP in Ramos cells and imaged its localization in cells forming immune synapses with anti-IgM-coated plasma membrane sheets. Endophilin A2 formed highly dynamic spots in the synapse that co-localized with antigen clusters (Fig 2F, Video 1). Furthermore, imaging of endophilin A2-GFP and mCherryclathrin light chain suggested that endophilin A2 works independently of CCPs as the majority of endophilin A2-GFP spots did not colocalize with CCPs labeled with mCherry-clathrin (Fig 2F). Quantification confirmed that only $17.44 \%$ of endophilin A2 clusters overlapped with CCPs and only $15.65 \%$ of CCPs overlapped with endophilin A2 spots, only slightly higher than colocalization in randomized controls (Fig 2G). In addition, live cell imaging showed that the instances of endophilin A2 and CCP overlap did not have a specific temporal pattern and that endophilin A2-GFP spots interacted with antigen clusters without prior recruitment of clathrin (Video1). These observations are in line with a clathrin-independent role of endophilin A2 in FEME.

To understand how endophilin A2 is recruited to activated BCRs, we analyzed endophilin A2 complexes in anti-IgM-stimulated Ramos cells using a BioID2 proximity labelling assay ${ }^{31}$ (Fig S2A). This assay captured known interaction partners of Endophilin A2, including dynamin2 (DNM2); intersectin2 $(I T S N 2)^{32}$ and Alix $(P D C D 6 I P)^{33}$. Among the detected interaction partners were also BCR 
signaling adaptors GRB2 and BLNK (Fig S2B, Supplementary table 4). To test the importance of these adaptors, we used pulldown assays to analyze interactions of endophilin A2-GFP with the BCR in DG75 Burkitt lymphoma cells in the context of GRB2 or BLNK deletion ${ }^{34}$. Similar to Ramos cells, endophilin A2-GFP was eluted in the anti-IgM-interacting fraction in WT DG75 cells (Fig 2H). The amount of endophilin A2-GFP was markedly reduced in anti-IgM pulldown in the absence of GRB2 and also slightly reduced in the absence of BLNK. Furthermore, while we detected similar numbers of endophilin A2-GFP spots in synapses of GRB2 and BLNK-deficient DG75 cells compared to parental DG75 cells, deletion of GRB2 significantly reduced endophilin A2 colocalization with antigen clusters (Fig 2I). These results indicate that endophilin A2's recruitment to ligand-activated BCRs is regulated by complexes organized by the adaptor GRB2 with possible participation of BLNK.

\section{Endophilin A2 deletion results in abrogated development and function of mature B cells}

Endophilin A2 is the only endophilin A family member expressed in hematopoietic cells. To understand the role of Endophilin A2 in immune cell development and responses to antigens in vivo we developed a CRISPR gene targeting strategy in mouse hematopoietic stem cells (HSCs) followed by adoptive transfer to irradiated host mice (Fig 3A). We used HSCs from Cas9 knockin mice (expressing GFP) and targeted them with lentivirus encoding sgRNA and an mCherry marker. In the resulting chimeric mice, CRISPR-targeted cells were detected by flow cytometry and $\mathrm{mCherry}^{+}$percentage of the donor $\mathrm{GFP}^{+}$population was used to monitor cell development in the bone marrow and in the spleen. As a control, we used a $C d 4$-targeting sgRNA, which showed efficient abrogation of the development of mCherry ${ }^{+} \mathrm{CD} 4$-positive $\mathrm{T}$ cells, but did not affect mCherry ${ }^{+}$proportions throughout $\mathrm{B}$ cell development (Fig 3B). Thus, this strategy is efficient and specific for gene targeting in vivo and allows analysis of gene effects on B cell development and immune responses.

Targeting with an sgRNA specific for Sh3gll showed an efficient disruption of Sh3gll at the genomic level (Fig S3A) and a reduction of Sh3gll mRNA (Fig S3B). Sh3gll-targeted mCherry ${ }^{+}$B cell proportions in the bone marrow and in the transitional populations in spleen remained constant and similar to controls, indicating that early B cell development progressed normally in the endophilin A2deleted cells (Fig 3B). However, proportions of $\mathrm{mCherry}^{+}$cells declined to about half in mature 
follicular B cells and were 5-10 times reduced in marginal zone (MZ) B cells (Fig 3B, C). In comparison, mCherry proportions in T cells (Fig 3B) and other non-B cells (not shown) were not affected, indicating a selective role of endophilin A2 in B cells.

To investigate antigen-specific responses of $\operatorname{Sh} 3 g l l$-targeted B cells to immunization, we crossed Cas9 mice with $\mathrm{SW}_{\mathrm{HEL}}$ mice ${ }^{35}$ carrying B cell specificity for hen egg lysozyme (HEL). Bone marrow from the resulting progeny was used for CRISPR targeting and bone marrow chimeras. Following adoptive transfer of follicular B cells to C57BL/6 mice, we immunized with HEL conjugated to sheep red blood cells (SRBCs) and analyzed early activation, GC responses and generation of plasma cells (PCs). At day 1 after immunization, Sh3gll-targeted B cells upregulated activation markers CD69 and CD86 similarly to controls (Fig 3D, E). However, at days 4-14, they produced significantly fewer classswitched IgG1 cells, GC cells and PCs (Fig 3F). Thus, endophilin A2 is dispensable for antigen-induced B cell activation, but is required for GC responses and efficient generation of PCs.

\section{Endophilin A2 knockout mouse confirms B cell developmental defects and exhibits diminished}

\section{GC and antibody responses}

To confirm the importance of endophilin A2 in B cell responses, we characterized the immune system

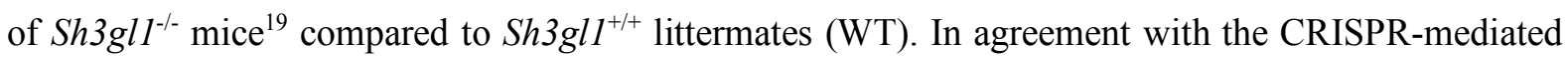
deletion, Sh3gl1/-- mice showed normal early B cell development in the bone marrow and in the transitional populations in the spleen but a reduction in splenic follicular and MZ B cells, as well as mature B cells in lymph nodes (Fig 4A). In contrast, numbers of $\mathrm{CD}^{+}$and $\mathrm{CD} 8^{+} \mathrm{T}$ cells in lymph nodes were normal. This B cell lymphopenia was reflected in reduced spleen size of $S h 3 g l l^{-/ 2}$ mice, compared to WT littermates (Fig 4B). In addition, Sh3gl1 $1^{-/-}$follicular B cells had an altered surface marker phenotype, similar to that obtained by CRISPR targeting (Fig 4C, and not shown).

To understand the role of endophilin A2 in antibody production, we immunized the mice with Tdependent antigen, NP-CGG. Sh3gll/- mice exhibited severely reduced total and NP-specific GC B cell numbers (Fig 4D). Quantification of total and NP-specific serum antibody levels showed that $S h 3 g l 1^{-/}$ 

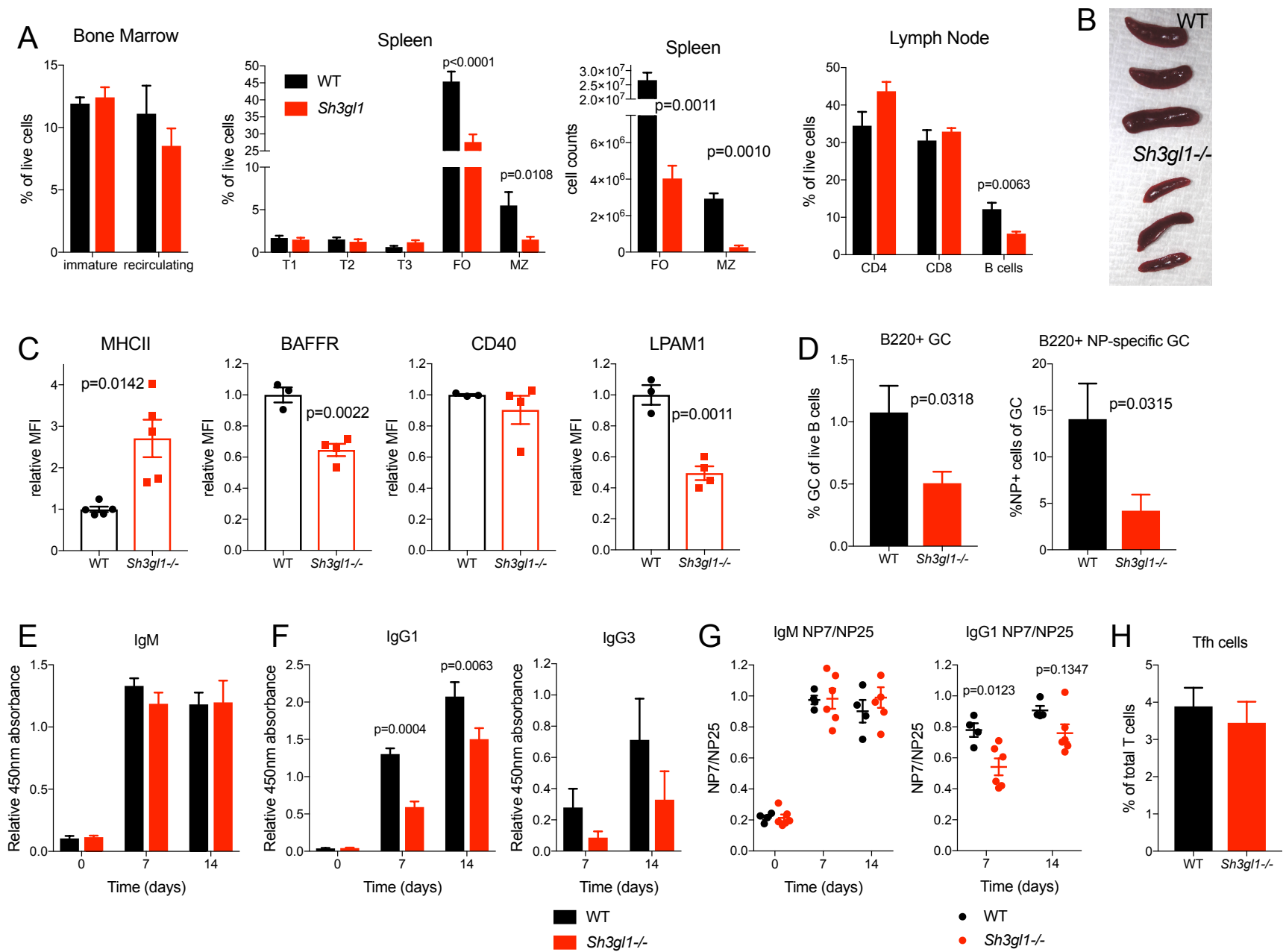

Figure 4. Endophilin A2 knockout mouse mimics the phenotype of CRISPR-targeted chimeras and exhibits reduced levels of affinity matured serum antibodies upon immunization. (A) Characterization of B cell populations in bone marrow, spleen and lymph node at steady state. Data show mean and SEM from $\mathrm{N}=5$ mice. $\mathrm{P}$, significance in $\mathrm{t}$ tests performed for follicular (FO) and MZ cell counts. (B) Spleen size in Sh3gll-/- and WT littermates at 12 weeks of age. (C) Surface levels of MHCII, BAFFR, CD40 and LPAM1 in Sh3gll-/- and WT littermates. P, statistical significance from unpaired $\mathrm{t}$ tests. (D) Quantification of $\mathrm{B} 220^{+} \mathrm{Fas}^{+} \mathrm{CD} 38^{-} \mathrm{GC}$ population as percentage of total splenic $\mathrm{B}$ cells and NP-specific cells as a percentage of the GC population 14 days after NP-CGG immunization in alum. Data show mean \pm SEM from $N=6$ mice, $p$, significance in unpaired $t$ tests. (E-F) Serum antibodies collected pre-immunization and 7or 14-days post NP-CGG immunization, detected by NP7-BSA ELISA using isotype-specific secondary antibodies. Data show mean $\pm \mathrm{SEM}$ from $\mathrm{N}=3$ experiments with statistical significance from 2-way ANOVA with multiple comparisons. (G) Ratio of binding to NP7 and NP25, as measured by ELISA. (H) Numbers of Tfh cells 14 days postimmunization, identified as a $\mathrm{CD} 4{ }^{+} \mathrm{CD} 44^{+} \mathrm{PD}-1^{+} \mathrm{CXCR} 5^{+}$population. Mean and $\mathrm{SEM}$ of $\mathrm{N}=4$ mice. 
mice had normal total baseline IgM and IgG levels (Fig S4), and mounted a relatively normal IgM response (Fig 4E). However, they showed markedly decreased serum NP-specific IgG1 and IgG3 at 7 and 14 days post-immunization (Fig 4F).

To investigate the effect of endophilin A2 knockout on affinity maturation we compared the binding of serum antibodies to low valency NP7 and high valency NP25 antigens. The NP7/NP25 binding ratio, used as a measure of affinity, increased in WT mice by day 7 post-immunization. While no differences were seen in the NP7/NP25 binding ratio of IgM in Sh3gl1 ${ }^{-/}$mice, the serum IgG ratio was significantly lower at day 7 and did not reach WT levels at day 14, suggesting inefficient antibody affinity maturation (Fig 4G).

These data, together with normal numbers of T follicular helper (Tfh) cells after immunization (Fig 4H) indicate a B cell-intrinsic role of endophilin A2 in GC B cell responses, class switching and affinity maturation.

\section{Endophilin $\mathrm{A} 2$ is dispensable for antigen presentation to $\mathrm{T}$ cells}

The GC defect could reflect an inability of the endophilin A2-deleted B cells to compete for T cell help as a result of lower BCR-mediated antigen uptake, processing and presentation. Follicular B cells isolated from the chimeric mice exhibited a small but consistent reduction in IgM BCR internalization (Fig 5A). A similar reduction was observed in $S h 3 g l 1^{-/}$mice (not shown). In contrast, internalization of IgD BCR appeared unaffected in the absence of endophilin A2 (Fig 5B). In addition, we observed a significant reduction of IgG-BCR internalization after anti-IgG stimulation of $S h 3 g l 1^{-/}$GC B cells (Fig 5C), confirming a role for endophilin A2 in IgM and IgG BCR endocytosis in both naïve and GC B cells. Unexpectedly, despite slower internalization after anti-IgM stimulation, both in solution (Fig 5A) and from PMS (Fig 5D), surface levels of IgM were lower in $S h 3 g l 1^{-/-}$B cells (Fig 5E). This could be explained by increased rates of degradation of surface IgM three hours after anti-IgM stimulation (Fig 5F, G), suggesting that while endophilin A2 positively regulates BCR endocytosis, it negatively regulates BCR delivery into degradative compartments.

To directly test the role of endophilin A2 in antigen presentation, we adoptively transferred HELspecific B cells from endophilin A2 HSC-targeted bone marrow chimeras into WT mice, followed by 
immunization with SRBC-HEL conjugated to Ea peptide, as previously described ${ }^{36}$. B cell antigen presentation was analyzed 1 day later by staining for E $\alpha$ peptide-MHCII complex on the cell surface. Sh3gll-targeted B cells exhibited similar antigen presentation, despite lower antigen uptake (Fig 5H, I). Similar results were obtained in vitro when isolated B cells were cultured with E $\alpha$ peptide conjugated to anti-Igא via a biotin-streptavidin bridge (Fig 5J). Furthermore, sorted mCherry ${ }^{+}$Sh $3 g l l$-targeted B cells pulsed with anti-Igא conjugated to ovalbumin stimulated normal proliferation of ovalbuminspecific OT-II CD4 T cells in co-culture assays (Fig 5K). Thus, despite the role in BCR internalization, endophilin $\mathrm{A} 2$ is not essential for $\mathrm{B}$ cell antigen presentation to $\mathrm{T}$ cells, possibly because it simultaneously limits degradative BCR trafficking and MHCII expression.

We also observed normal antigen-induced calcium flux (Fig S5A, B) and chemokine-induced migration (Fig S5C), suggesting that early functions required for B cell activation and interaction with $\mathrm{T}$ cells in vivo are not affected by endophilin A2 absence.

\section{Endophilin A2 is important for cell metabolic support of B cell growth and proliferation}

To understand if endophilin A2 is required for the response of B cells to $\mathrm{T}$ cell help, we analyzed proliferation of DDAO-labelled B cells stimulated by CD40L. We found a severely reduced ability of Sh3gll-targeted B cells to expand in response to CD40L compared to controls (Fig 6A). However, CD40 expression was normal and early transcription induced by CD40L was also unaffected in $S h 3 g l l^{-}$

I- B cells as evidenced by RNAseq (Fig 6B, S6A, Supplementary Table 5), suggesting a more downstream defect in cell expansion. Consistently, we observed that the defect in expansion was not limited to CD40L stimulation, but also occurred in response to BCR and TLR stimuli (Fig 6C). The reduced expansion was primarily a result of reduced proliferation, as rates of cell death in response to CD40, BCR and TLR ligation were not elevated (Fig 6D). However, cell survival in response to BAFF was reduced. These findings were reproducible in non-competitive B cell cultures from $\mathrm{Sh} 3 \mathrm{gll} \mathrm{I}^{-/-}$mice (Fig S6B, C), although results from IgM and TLR stimulation did not reach statistical significance under these conditions. In contrast, T cells isolated from these mice proliferated similarly to WT in response to anti-CD3 plus anti-CD28 stimulation (Fig S6B, C). 

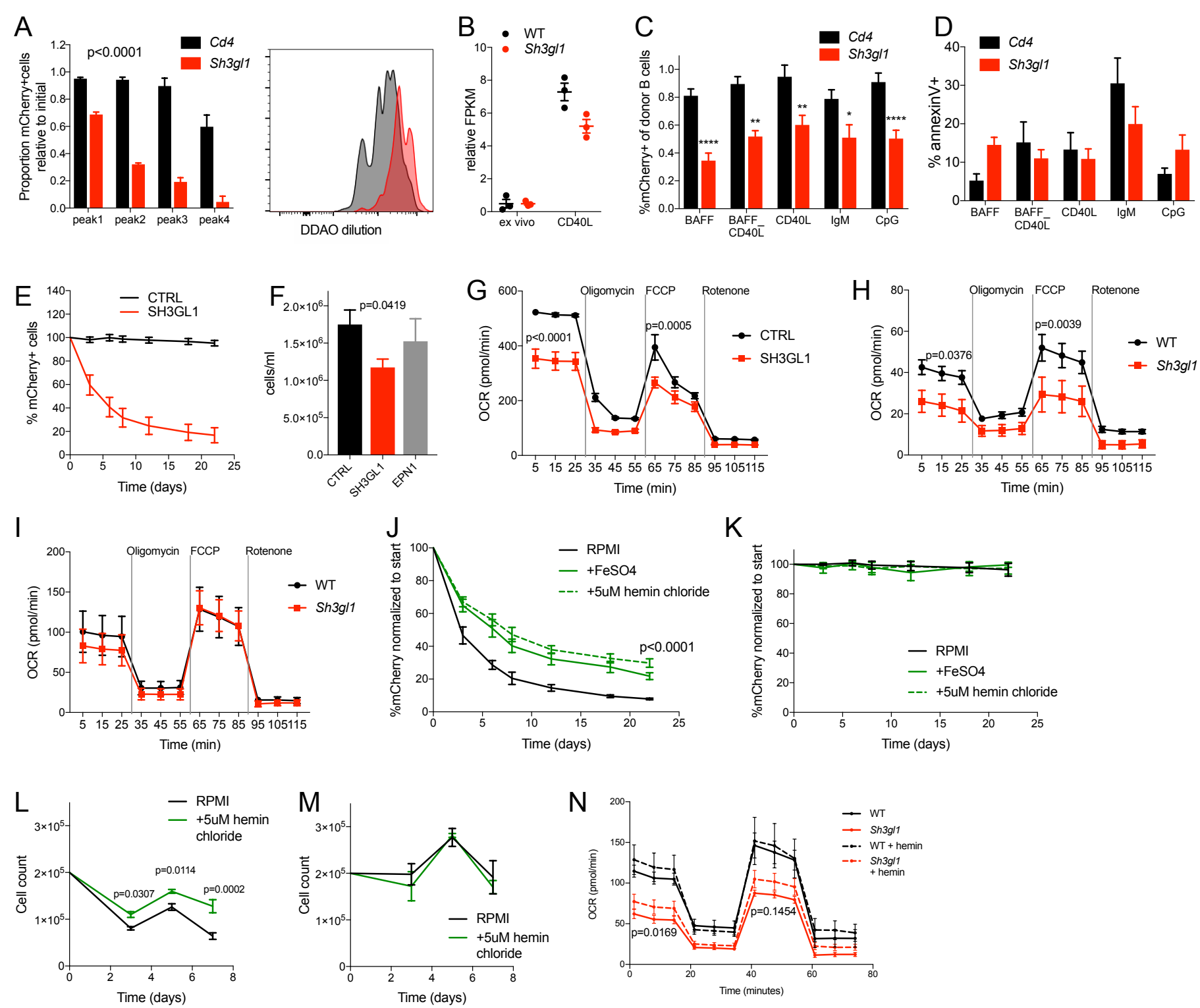

Figure 6. Endophilin A2 is required for B cell iron-dependent oxidative phosphorylation and cell expansion. (A) CRISPR-targeted follicular B cell proliferation after 3 days of CD40L stimulation. mCherry ${ }^{+}$populations were quantified within individual DDAO peaks. $\mathrm{N}=3$ mice. $\mathrm{P}$, statistical significance calculated by two-way ANOVA reached $\mathrm{p}<0.0001$ for all proliferation peaks. (B) CD80 mRNA levels in B cells following 24 hours of CD40L culture, relative to untreated B cells. Data from RNAseq of $N=3$ mice. (C-D) Isolated naïve B cells from CRISPRtargeted chimeras after 3-day culture in stated cytokines. (C) mCherry percentage in targeted primary cell cultures. $\mathrm{N}=15$ mice. ${ }^{*} \mathrm{p}<0.05,{ }^{*} \mathrm{p}<0.01, * * * * \mathrm{p}<0.0001$ using two-way ANOVA. (D) AnnexinV surface stain in targeted cells. $\mathrm{N}=3-4$ mice. (E) mCherry ${ }^{+}$cell percentage in CRISPR-targeted Ramos cells over time. $\mathrm{N}=6$ independent experiments. (F) Cell counts at passage 3-6 of CRISPR-targeted puromycin-selected Ramos cells. P, statistical significance from unpaired $t$ test. $(\mathrm{G})$ Oxygen consumption rate (OCR) in CRISPR-targeted Ramos cells. P, two-

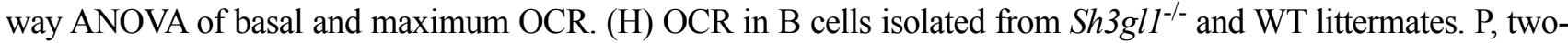
way ANOVA of basal and maximum OCR. (I) OCR in T cells isolated from Sh $3 g l l^{-/-}$and WT littermates. (J-M) Cell cultures with full RPMI media supplemented with $50 \mu \mathrm{g} / \mathrm{ml} \mathrm{FeSO}_{4}$ or $5 \mu \mathrm{M}$ hemin chloride. (J) SH3GL1targeted Ramos cells. $\mathrm{N}=4$ independent infections. P, statistical significance from two-way ANOVA from both treatments versus RPMI. (K) Control sgRNA-targeted Ramos cells. (L) Sh3gl1 $1^{-/-}$isolated B cells, maintained in CD40L culture. $\mathrm{N}=3$ mice. P, significance calculated by two-way ANOVA. (M) B cells isolated from WT littermates, maintained in CD40L culture. (N) OCR in Sh3gll ${ }^{-/}$or WT B cells after overnight culture in CD40L, supplemented with $5 \mu \mathrm{M}$ hemin chloride. $\mathrm{N}=6$ mice. $\mathrm{P}$, two-way ANOVA with matched samples for basal and maximum OCR of Sh3gll compared to Sh3gll + hemin. All data show means \pm SEM. 
Analysis of CRISPR survival scores determined in Ramos cells using the Brunello library also indicated cell-intrinsic essentiality of endophilin A2 in growth (Fig S6D). In addition, re-analysis of published CRISPR screens ${ }^{30,37,38}$, indicated selective requirement for endophilin $\mathrm{A} 2$ for growth of $\mathrm{B}$ cell lymphoma lines, particularly Burkitt and GCB diffuse large B cell lymphoma types, but not the diffuse large $\mathrm{B}$ cell $\mathrm{ABC}$ type or other hematopoietic lines (Fig S6D).

In Ramos cells, CRISPR-mediated SH3GL1 targeting confirmed a progressive loss of the targeted $\left(\mathrm{mCherry}^{+}\right)$population over time (Fig 6E), which was further exacerbated with increased levels of competition (Fig S6E). This cell intrinsic defect was also apparent in puromycin-selected cultures as reduced cell numbers at each passage and was specific to endophilin A2 as compared to CCP components (Fig 6F). Using a BrdU pulse-chase assay in SH3GL1-targeted Ramos cells, we established that the reduced cell expansion was not a result of a block at a specific cell cycle checkpoint, but an overall slower rate of cell cycle progression (Fig S6F).

To identify the reason for the B cell growth defect, we performed RNA sequencing on sorted follicular B cells from WT or $S h 3 g l 1^{-/-}$mice ex vivo or post-CD40L activation. Gene set enrichment analysis in the absence of endophilin (GSEA, Fig S6G) revealed downregulation of pathways regulating cell activation, vesicle-mediated transport, endocytosis, protein transport and signal transduction. Interestingly, gene sets upregulated in the absence of endophilin included many metabolic processes, cell cycle and organization of mitochondria, ER and the plasma membrane. To investigate possible role of cell metabolism and mitochondrial function, we measured oxygen consumption rate (OCR) in Sh $3 g l 1^{-/}$primary B cells and CRISPR-targeted Ramos cells. Endophilin A2-deficient cells exhibited reduced basal and maximal respiration compared to controls, consistent with metabolic impairment highlighted in the transcriptional analysis (Fig 6G, H). Contrastingly, OCR of T cells isolated from Sh $3 g l l^{-/-}$mice were similar to WT T cells (Fig 6I).

The transcriptional analysis also revealed significant enrichment (at FDR $<25 \%$ ) of several iron and

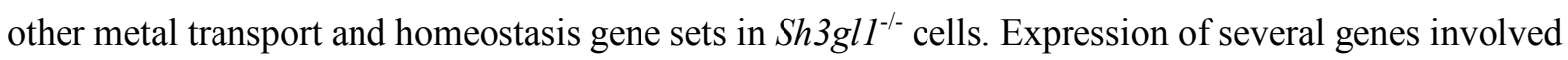
in regulating iron ion homeostasis was elevated in Sh3gll $1^{-/-} \mathrm{B}$ cells (Fig S6H). Given the importance of cellular iron homeostasis for mitochondrial respiration and lymphocyte proliferation ${ }^{39,40}$, we 
endeavored to test whether iron uptake played a role in the metabolic and survival defects in the absence of endophilin A2. Iron supplementation using ferrous sulphate or hemin chloride in both Ramos and primary B cell cultures showed a consistent though incomplete rescue of cell growth over time (Fig 6J, L), with little effect on non-targeted or WT cells (Fig 6K, M). In contrast to iron rescue, SH3GL1deleted Ramos cells were not rescued by supplementation with glucose, glutamine or non-essential amino acids (NEAA) (Fig S7A, B). Glucose uptake in SH3GL1-targeted Ramos cells was also normal (Fig S7C, D) and growth defects were apparent in the context of normal mitochondrial mass (Fig S7E, F). Furthermore, iron supplementation resulted in a partial rescue of cellular OCR in the Sh3gl1 ${ }^{-/-}$B cells with little effect on WT cells (Fig 6N). As this supplementation bypasses the requirement for transferrin receptor (TFRC) internalization, we analyzed transferrin uptake in CRISPR-targeted Ramos and primary naïve or GC B cells. In Ramos, SH3GL1-targeting reduced TFRC internalization similar to PICALM depletion (Fig S7G). This reduction was recapitulated in naive $S h 3 g l l^{-/}$B cells and was particularly dramatic in GC B cells (Fig S7H, I). Together these results highlight a B cell-specific requirement for endophilin A2 in cellular metabolism, growth and expansion, in part by regulating iron uptake.

\section{DISCUSSION}

Our study presents a large-scale, unbiased characterization of antigen internalization in B cells enabled by whole genome CRISPR screening. We have validated 72 genes positively and negatively regulating BCR internalization, covering a wide range of cellular processes. As expected, the screen hits included CCP components, such as EPN1 and PICALM, confirming the role of CME and pointing to a specific subset of CCP components that are the most intimately involved in antigen uptake. The screen also identified a number of genes regulating endosomal maturation and lysosome biology, suggesting tight coupling of BCR endocytosis to downstream trafficking pathways. Most of the identified endocytic genes are predicted to have ubiquitous roles, but we also captured proteins known to participate in BCR signaling specifically, such as Cbl. The importance of BCR-specific pathways interacting with general endocytic and trafficking proteins is supported by previous observations of BCR signaling inducing phosphorylation of CCP components ${ }^{10}$ as well as endosomal proteins, such as the orchestrator of 
endosomal maturation RAB7A, one of the most highly phosphorylated proteins in B cells after antigen binding ${ }^{15}$. Studies of B cell-specific regulation of the common endocytic proteins could expand our analysis in the future.

Despite the inherent noise associated with whole genome approaches, our data illustrate that pooled library CRISPR screening has matured into a tool that surpasses previous approaches in efficiency and robustness. Remaining limitations for functional screens, such as ours, are gene redundancy and lethal effects of targeting genes essential for cell growth and survival. However, our screens still captured candidates that are partly redundant, such as EPN1, and genes that are also essential for growth, such as endophilin A2. Nevertheless, the requirement of endophilin A2 for B cell growth did prevent its identification as an endocytic regulator in one of our screens using the Brunello library, with subsequent analysis validating that this was due to strong depletion of SH3GL1 targeting sgRNA sequences from the cell population before the endocytic assay. Improved screening approaches in the future can further increase sensitivity of these genome-wide assays, and allow more sophisticated screening for example to involve immune synapse formation. Our results show the potential of such discovery science, paving the way for other whole-genome studies in immune cells.

This work led to the identification of endophilin A2 as a novel regulator of antigen uptake in B cells. Our results are in line with the described role of endophilin A2 in $\mathrm{FEME}^{21}$, highlighting a rapid, antigentriggered and clathrin-independent route of BCR endocytosis. Although our data do not completely rule out the participation of endophilin A2 in CME suggested by earlier studies ${ }^{19,41,42}$, the distinct localization of endophilin A2 in B cell synapses and the selective effect on antigen-induced BCR internalization contrast with CME and suggest that endophilin A2 in B cells acts predominantly in a separate, clathrin-independent pathway.

We also identified GRB2 as an important adaptor in the antigen-dependent recruitment of endophilin A2 to the BCR. Antigen ligation of the BCR leads to activation of Syk and Src family kinases, tyrosine phosphorylation of the BCR and formation of large signaling complexes nucleated by adapter proteins including BLNK and GRB2. The molecular mechanisms by which GRB2 regulates endophilin A2 recruitment will however require further studies. GRB2 contains one SH2 and two SH3 domains, but 
no direct proline-rich motif to bind endophilin A2's SH3 domain, making it unlikely that it recruits endophilin A2 directly. Instead, GRB2 may stabilize recruitment of endophilin A2 through other interactions, which themselves may be redundant. Candidates from our BioID2 assays include BLNK and ITSN2 ${ }^{43}$. BLNK in particular was partly required for endophilin A2 recruitment to the BCR in our experiments. ITSN2 has recently been described to be important for B cell expansion during immune responses $^{44}$ and interacts with GRB2, providing a possible additional link between endophilin and BCR signaling complexes.

Despite regulating antigen endocytosis, endophilin A2 was dispensable for B cell presentation of antigenic peptides on MHC II. It is possible that contribution of FEME to BCR trafficking to MHC II processing compartments is sufficiently compensated by the eventual delivery of antigen into these compartments by CME. Alternatively, FEME may actively route the BCR away from processing compartments. This is supported by our finding that the IgM BCR is degraded faster after antigen binding in endophilin A2-deficient B cells. In addition, defects in antigen processing may be compensated by the dramatically enhanced expression of MHC II on the surfaces of endophilin A2deficient B cells, the basis of which remains to be investigated. Although our data cannot currently exclude a selective antigen presentation defect in GC B cells, we favor the explanation that the impairment of GC B cell responses in endophilin A2-deficient mice is a consequence of the essential role of endophilin A2 in B cell-intrinsic growth and proliferation.

The loss of endophilin A2 also resulted in severe disruption of the MZ, an area of the spleen crucial for rapid humoral responses to blood-borne pathogens. This loss may be a result of a developmental block or impaired maintenance caused by abnormal BCR, BAFFR, Notch, integrin or chemokine receptor functions ${ }^{45}$. Indeed, we have detected lower IgM BCR surface levels, and reduced BAFFR and integrin expression. However, we could not attribute the MZ defects to a specific BCR signaling defect; and Notch target gene expression and chemokine-induced chemotaxis were also normal. It is thus possible that reduced MZ B cell development and maintenance are associated with reduced BAFFR functions, observed in survival assays in vitro. Lower BAFFR function may also contribute to lower numbers of follicular B cells. Alternatively, the reduction in MZ B cells may reflect as yet unknown endosomal trafficking requirements specific for the MZ. Interestingly, dysregulation of MZ B cells observed upon 
loss of other endocytic regulators, such as the clathrin adaptor, EPS15 which negatively regulated antigen uptake in our screen, has been shown to lead to a significant increase in MZ B cell numbers ${ }^{46}$. We have shown that endophilin A2 is crucially required for the final stage of development or maintenance of mature B cells and their response to immunological challenge. In the absence of endophilin A2 we observed reduced numbers of GC B cells, lower numbers of PCs, lower antigenspecific serum IgG1 and IgG3 antibody levels, and impaired IgG affinity maturation. This, together with normal early signaling and activation of B cells in vitro and in vivo points to a defect in B cell expansion at the onset and during the GC reaction. Given the absence of effects on MHC II-mediated antigen presentation discussed above, the defect is best explained by the essentiality of endophilin A2 in cell-intrinsic growth of CD40-activated B cells and GC-like B cell lymphoma cell lines. Further investigation of the defect showed that the impaired growth was associated with reduced mitochondrial respiration capacity. Mitochondrial respiration and cell growth defects were not associated with reduced glucose uptake and could not be rescued by supplementation with amino acids. Instead we found that supplementation with free iron partially rescued both mitochondrial respiration and cell growth in the Ramos cell line and activated primary mouse B cells. These results strongly implicate impaired iron uptake in the defect.

Iron has been increasingly linked to efficient immune defenses. Both nutritional iron deficiency and genetic defects affecting cellular iron uptake cause immunodeficiency in humans. The role of iron in the immune system has been particularly strongly linked to lymphocyte proliferation in response to antigen ${ }^{40,47-49}$. Our data provide a novel insight into the unique regulation of iron uptake in proliferating GC B cells. It is possible, that in GC B cells, endophilin A2 directly participates in endocytosis of TFRC. Indeed, we have observed reduced TFRC endocytosis in endophilin A2-deficient GC B cells. These data contrast with the reported lack of effect of endophilin A2 knock down on TFRC endocytosis in $\mathrm{T}$ cells $\mathrm{s}^{21}$ and our results showing normal proliferation of endophilin A2-deficient $\mathrm{T}$ cells. Thus, the role of endophilin A2 in TFRC endocytosis is GC B cell specific and possibly related to the unique transcriptional profile of GC B cells, which includes changes in expression, localization and function of endocytic and trafficking proteins ${ }^{50}$. However, it is unlikely that the growth defect in endophilin A2 deficient B cells is mediated solely by reduction in TFRC endocytosis. This interpretation comes from 
our observation that deletion of CCP components EPN1 or PICALM, which reduced TFRC endocytosis similarly to deletion of endophilin A2 did not result in altered cell metabolism or growth. Thus, endophilin A2 may have roles in iron uptake that extend beyond TFRC endocytosis and may include effects on subsequent TFRC trafficking. Given that iron homeostasis is crucial across all hematopoietic lineages $^{40}$, all of which express endophilin A2 as the only endophilin A family member, the selective defect caused by endophilin A2 deletion in GC B cells thus points to a specialized regulation of endocytosis and iron uptake at this stage of the immune response. Such regulation may use the signaling-dependency of endophilin A2's function to couple iron uptake to signals driving positive selection of GC B cells. This interpretation is consistent with the importance of endophilin A2 for affinity maturation of the antibody response.

In summary, we have shown a novel B cell-intrinsic role for endophilin A2 in antibody responses. The critical role of endophilin A2 in B cell growth depending on trace element uptake is enhanced by cellular competition and may thus support rapidly dividing B cells in low-nutrient conditions. In the light of the partial rescue of the growth defect by iron supplementation, other nutrients may become limiting as well. This unique regulation of nutrient uptake is important in protective $\mathrm{GC}$ responses but also in B lymphoma cells derived from the GC as indicated by published genome-wide screens, in which endophilin A2 appears to be essential selectively in cell lines derived from Burkitt and diffuse large B cell lymphomas of the GC-type. Thus, endophilin-regulated intracellular trafficking has novel implications for B cell immunity and pathology through regulation of antigen uptake, endocytic homeostasis and cell metabolism.

\section{METHODS}

\section{Mice and cell isolation}

C57BL/6 and Cas9 mice on a C57BL/6 background were used as a sources of primary mouse B cells. In addition, mice were crossed with $\mathrm{SW}_{\mathrm{HEL}}\left(\operatorname{Igh}^{\mathrm{tm} \mid \mathrm{Rbr}}-\mathrm{Tg}(\operatorname{IgkHyHEL10}) 1 \mathrm{Rbr}\right)$ mice $^{35}$. To generate bone marrow chimeras, C57BL/6 mice were lethally irradiated with 2 doses of 5Gy and reconstituted with bone marrow by intravenous injection (100 000 cells/ host). For in vitro B cell studies, naïve murine B cells were isolated by negative selection using anti-CD43 microbeads (Miltenyi). All mice were bred 
and treated in accordance with guidelines set by the UK Home Office and the Francis Crick Institute Ethical Review Panel.

\section{Stable cell lines and lentiviral transduction}

Ramos cells were maintained in full RPMI (10\% fetal calf serum (FCS, BioSera), $100 \mu \mathrm{M}$ non-essential amino acids, $20 \mathrm{mM}$ HEPES, $2 \mathrm{mM}$ glutamine, $50 \mu \mathrm{M}$ beta-mercaptoethanol). DG75 cells were sourced from the Human Collection for Microorganisms and Cell Cultures in Braunschweig and maintained similarly.

To create stable Ramos Cas9 cells, recombinant replication-incompetent lentiviruses were produced by co-transfecting HEK293T cells using transIT-LT1 (Mirus) with pMD2.G and psPAX2 helper plasmids together with lentiCas9-Blast (Addgene, \#52962). Lentivirus was harvested 48- and 72-hours following transfection, concentrated by ultracentrifugation and used to spin-infect Ramos cell lines for 90 min at $1350 \mathrm{x} \mathrm{g}$ in the presence of $10 \mu \mathrm{g} / \mathrm{ml}$ polybrene (Sigma). Forty-eight hours after the spinfection, cells were selected using $10 \mu \mathrm{g} / \mathrm{ml}$ blasticidin. Cells were single cell sorted and expanded.

\section{CRISPR-mediated gene disruption}

CRISPR sgRNA sequences were designed using the Broad Institute's sgRNA Designer. Forward and reverse oligonucleotides including the guide sequence were synthesized, phosphorylated, annealed and individually cloned into lentiGuide-Puro or lentiGuide-mCherry (in which puromycin resistance cassette was replaced by mCherry) plasmids. Lentivirus was produced and cells spinfected as described above. Cells were selected using $2.5 \mu \mathrm{g} / \mathrm{ml}$ of puromycin. CRISPR-targeted cells were used within two weeks of selection. Cells expressing a fluorescent marker were further purified using Avalon cell sorter (Propel Labs).

\section{CRISPR Screens and analysis}

GeCKO and Brunello whole-genome human libraries were acquired from Addgene. Smaller scale validation library was created by selecting the top 300 positive and 50 negative regulators from the Brunello screen and filtering for expression in Ramos cells; non-targeting and essential gene controls were added to the 213 experimental genes.

Stable Ramos Cas9 cells were expanded and $80 \times 10^{6}$ cells spinfected with pooled lentiviral wholegenome CRISPR libraries at multiplicity of infection of 0.5 , achieving 500x library coverage. This was appropriately scaled down for custom library infections. A sample of the infected cells was removed for genomic DNA analysis; the rest were selected with $2.5 \mu \mathrm{g} / \mathrm{ml}$ of puromycin for 3 weeks and maintained at 1000x library coverage at every passage. Internalization assays were performed as described below. Sorted "internalized" and "non-internalized" populations, as well as whole populations pre- and post-selection were used to extract gDNA (DNeasy Blood \& Tissue kit, Qiagen) and amplify the integrated viral genome using a nested PCR, as described in Shalem et $a^{51}$. Libraries 
were sequenced on a MiSeq system (Illumina). Raw reads in demultiplexed FASTQ files were trimmed to contain sgRNA sequences only and these were aligned to the original library using Bowtie. The number of aligned reads for each unique sgRNA was counted and used to calculate survival and internalization scores. Analysis was performed in MATLAB (MathWorks) and statistics were calculated using MAGeCK ${ }^{52}$.

Internalisation score $=\log 2\left(\frac{1+\text { sgRNA abundance non-internalised population }}{1+\operatorname{sgRNA} \text { abundance internalised population }}\right)$

\section{Surrogate antigens}

Model antigens used in solution or on PMS were goat anti-mouse Igא F(ab') ${ }_{2}$ (Southern Biotech) for mouse splenic B cells and goat $\mathrm{F}\left(\mathrm{ab}^{\prime}\right)_{2}$ anti-human Fc5 $\mu$ (Jackson Immunoresearch) for Ramos and DG75 cells. The antibodies were biotinylated using EZ-Link NHS-LC-LC-biotin (Pierce) and conjugated to one of several fluorophores - Cy5 Monoreactive dye (GE Healthcare), AlexaFluor 405 or AlexaFluor 647 NHS esters (ThermoFisher) in Sodium Carbonate buffer, according to manufacturer instructions. Excess dye was removed using Zeba 7K MWCO desalting columns (Pierce, ThermoFisher). Mouse transferrin (Rockland) was also fluorescently labelled and biotinylated for TFRC internalization assays.

\section{Soluble antigen internalization assay}

Murine B cells or Ramos cells were stained with a LIVE/DEAD marker then washed and incubated with the surrogate antigens described above at $2 \mu \mathrm{g} / \mathrm{ml}$ in PBS- BSA (1\%) for $10 \mathrm{~min}$ on ice. Cells were washed and incubated on ice or at $37^{\circ} \mathrm{C}$ for a set time frame $(5-30 \mathrm{~min})$ before fixation in $4 \%$ PFA. Washed cells were then stained for $30 \mathrm{~min}$ on ice with fluorescently labelled streptavidin, to counterstain remaining surface antigen. Samples were analyzed on a BD LSRFortessa. Internalization was quantified as the percentage of antigen remaining at the cell surface (streptavidin-labelled) using FlowJo (TreeStar). For large-scale screening assays, a minimum of 120 million cells was processed as described and highest and lowest internalizing populations (top and bottom 10-15\%) were sorted on a BD FACSAria.

\section{Live and fixed cell imaging}

Epifluorescence and TIRF imaging were carried out on a Nikon Eclipse Ti microscope with an ORCAFlash 4.0 V3 digital complementary metal-oxide semiconductor (CMOS) camera (Hamamatsu Photonics) and 100x TIRF objective (Nikon). Illumination was supplied by 405, 488, 552 and $637 \mathrm{~nm}$ lasers (Cairn) through an iLas ${ }^{2}$ Targeted Laser Illuminator (Gataca Systems) which produces a $360^{\circ}$ spinning beam with adjustable TIRF illumination angle.

Plasma membrane sheets (PMS) were generated as described previously ${ }^{53}$ in 8-well Lab-Tek imaging chambers (Thermo). Isolated primary B cells or Ramos cells were washed and added to pre-warmed 
imaging wells; allowed to interact with antigen-loaded PMS at $37^{\circ} \mathrm{C}$. Cells were imaged live or fixed in $4 \%$ PFA after $20 \mathrm{~min}$.

Acquired datasets were analyzed using automated routines in ImageJ and MATLAB (MathWorks) All cells imaged were included in the analyses. Substrate-bound antigen internalization was quantified by detecting antigen clusters extracted from the PMSs inside the B cells identified using B220 or CD19 surface staining as described previously ${ }^{54}$. Endophilin A2-GFP and clathrin-mCherry spots were detected as described ${ }^{55}$. Briefly, Endophilin A2 or clathrin TIRF images were enhanced by convolution with a $325 \mathrm{~nm}$-sized bandpass filter and spots above threshold were tracked and analyzed for fluorescence intensities in all relevant channels. Thresholds were set automatically as a fraction of each cell's mean fluorescence intensity. To analyzed Endophilin A2-clathrin colocalization, coinciding spots were detected as those whose centers were less than $128 \mathrm{~nm}$ apart. Random spot colocalization was calculated after randomly picking same numbers of non-overlapping spots from each cell's area.

\section{Antibodies and flow cytometry}

Erythrocyte-lysed single-cell suspensions were blocked with anti-CD16/32 for 15 min and stained with appropriate antibodies for $30 \mathrm{~min}$ on ice. The following stains and antibodies were used for immunophenotyping: B220 (RA3-6B2), CD25 (M-A251), CD69 (H1.3F2), CD86 (GL1) and CD95 (Jo2) from BD Bioscience; CD19 (1D3), CD23 (B3B4), CD38 (90), CD93 (AA4.1), IgM (II/41) and MHCII (M5/114.15.2) from eBioscience; CD21/CD35 (7E9), CD45.1 (A20), CD138 (281-2) and c-kit (2B8) from BioLegend.

In spleen, follicular and marginal zone cells were detected based on CD23 and CD21/35 expression in the mature cell population $\left(\mathrm{B} 220^{+} \mathrm{CD} 93^{-}\right)$. Transitional populations were detected in the immature population $\left(\mathrm{B} 220^{+} \mathrm{CD} 93^{+}\right)$based on expression of CD23 and IgM. Germinal centre cells were defined as $\mathrm{B} 220^{+} \mathrm{GL}^{+} \mathrm{Fas}^{+} \mathrm{CD} 38^{-}$population; plasma cells were detected by expression of $\mathrm{CD} 138$. In the bone marrow, early B cell populations were defined as follows: pre-pro B (B200 $\left.{ }^{+} \mathrm{c}-\mathrm{kit}^{+} \mathrm{CD} 25^{-} \mathrm{CD} 19^{-}\right)$, pro-B $\left(\mathrm{B} 200^{+} \mathrm{c}-\mathrm{kit}^{+} \mathrm{CD} 25^{-} \mathrm{CD} 19^{+}\right)$and pre-B cells $\left(\mathrm{B} 200^{+} \mathrm{c}-\mathrm{kit}^{-} \mathrm{CD} 25^{+} \mathrm{CD} 19^{+}\right)$.

\section{Western blot, immunoprecipitation and IgM degradation assay}

For signaling assays, a minimum of $5 \times 10^{6}$ cells were activated with soluble model antigens at $37^{\circ} \mathrm{C}$ for set time points, then washed and processed on ice. Cells were lysed in RIPA buffer (Sigma), containing cOmplete EDTA-free protease inhibitor cocktail (Roche), for $10 \mathrm{~min}$ on ice and then centrifuged at $16000 \mathrm{~g}$ for $10 \mathrm{~min}$ at $4^{\circ} \mathrm{C}$. Samples were boiled in 1X NuPAGE LDS Sample Buffer (Invitrogen) and 1X NuPAGE Sample Reducing Buffer (Invitrogen) at $95^{\circ} \mathrm{C}$ for $5 \mathrm{~min}$ and then separated using ExpressPlus PAGE Gels 4-20\% (GenScript). Proteins were transferred to a polyvinylidene fluoride (PVDF) membrane and blocked for 1 hour in PBST with 50\% Odyssey Blocking Buffer (LI-COR). Primary antibodies were incubated overnight in the same blocking solution; fluorescently-tagged 
secondary antibodies were incubated for 1 hour. Membranes were imaged using an Odyssey CLx or Odyssey Fc imaging systems (LI-COR).

For immunoprecipitation, cell lysates were incubated with biotinylated pull-down antibodies and streptavidin-M280 Dynabeads (Invitrogen) overnight at $4^{\circ} \mathrm{C}$. Beads were washed on a Dynabead magnet 5 times and eluted in 1x NuPAGE Sample Reducing Buffer at $95^{\circ} \mathrm{C}$. Samples were processed as for western blot above.

For IgM degradation assays, $30 \times 10^{6}$ primary mouse cells were surface biotinylated using EZ-Link Sulfo-NHS-LC-LC-biotin (Pierce) as per manufacturer instructions. After labelling, cells were washed in PBS with 100mM glycine to quench and remove excess biotin. Cells were washed 3 times and incubated with anti-IgM on ice for $15 \mathrm{~min}$. After washing, cells were incubated at $37^{\circ} \mathrm{C}$ for $0,3,6$ or 12 hours to allow internalization and recycling or degradation of surface proteins. Cells were then lysed; biotinylated proteins were pulled down using streptavidin-M280 Dynabeads and both supernatants and eluates processed for western blot.

\section{Gene expression analysis}

RNA was isolated from sorted follicular B cells, ex vivo or after overnight CD40L culture, using RNeasy mini kit (Qiagen). Poly-A enriched libraries were prepared using SMART-Seq HT and NexteraXT (Illumina) and sequenced on Illumina HiSeq 2500. Reads were aligned to C57BL/6J mouse reference genome mm10, GRCm38 and quantified using Tophet and Cufflinks.

\section{BrdU pulse-chase}

CRISPR-targeted Ramos cells were loaded with BrdU (Sigma) at final concentration of $10 \mu \mathrm{M}$ in full RPMI at $37^{\circ} \mathrm{C}$ for 1 hour. Cells were washed and cultured at $37^{\circ} \mathrm{C}$. They were harvested and processed at various time points to follow the labelled cohort through the cell cycle. Fixation was performed in cold $70 \%$ ethanol for 30 minutes with regulator vortexing. Samples were washed in PBS+ $2 \%$ BSA, resuspended in $500 \mu \mathrm{l}$ of $2 \mathrm{M}$ hydrochloric acid and incubated for $20 \mathrm{~min}$. Samples were washed 3 times to remove acid traces and labelled sequentially with anti-BrdU antibody (Becton Dickinson) at 1/50 dilution in PBT-tween for 30min; goat anti-mouse secondary antibody (AF488, Invitrogen) for 20min; and anti-MPM2-Cy5 antibody (EMD Millipore) at 1/200 dilution for 1hour, with thorough washing between stains. Finally, samples were stained with propidium iodide and analyzed by flow cytometry. Mitotic cells were detected as MPM2-positive; G1, S and G2 were identified by DNA content (PI). BrdU+ proportion was quantified within each population at given timepoints.

\section{Antigen presentation assays}

To detect antigen presentation in vitro, B cells were incubated with microbeads (Bangs laboratories) conjugated to anti-Igא and Ea-peptide (Biotin-GSGFAKFASFEAQGALANIAVDKA-COOH) for 5hours at $37^{\circ} \mathrm{C}$, fixed in $4 \%$ PFA and blocked with $2 \%$ BSA and Fc block. 
For in vivo assays, mice were immunized (intraperitoneal) with SRBC-HEL conjugated to Ea peptide, as previously described ${ }^{36}$. Splenocytes were harvested 24 hours later and blocked with $2 \%$ BSA and Fc block on ice. For both assays, samples were then stained with antibody against Ea52-68 bound to I-Ab (eBioY-ae, eBioscience) at 1/100 dilution in PBS-BSA.

\section{Cytokine cultures and iron supplementation}

To study proliferation, B cells were labelled with $1 \mu \mathrm{M}$ DDAO (Thermo) and cultured in full RPMI supplemented with $4 \mu \mathrm{g} / \mathrm{ml} \mathrm{CD40L} \mathrm{(R \& D} \mathrm{systems),} \mathrm{100ng/ml} \mathrm{BAFF} \mathrm{(Peprotech),} 1 \mu \mathrm{g} / \mathrm{ml}$ anti-IgM (Jackson Immunoresearch) or $2.5 \mu \mathrm{g} / \mathrm{ml} \mathrm{CpG} \mathrm{(Invitrogen).} \mathrm{DDAO} \mathrm{dilution} \mathrm{(and} \mathrm{mCherry} \mathrm{expression} \mathrm{in}$ CRISPR-targeted B cells) was detected by flow cytometry 3 days post-culture.

For cell expansion studies, full RPMI was supplemented with one of the following: $5 \mu \mathrm{M}$ hemin chloride (Sigma), $50 \mu \mathrm{g} / \mathrm{ml}$ ferrous sulphate (Sigma), $10 \mathrm{~g} / \mathrm{L}$ D-Glucose (Gibco), $10 \mathrm{mM}$ L-Glutamine (Gibco) or 5x non-essential amino acids (NEAA, Gibco).

\section{Calcium}

Cells were loaded with Fluo-4 and Fura-Red as per manufacturer instructions for $30-45 \mathrm{~min}$ a $37^{\circ} \mathrm{C}$ in RPMI $+5 \%$ FCS. Cells were washed and analyzed by flow cytometry in HBSS- $0.1 \%$ BSA. At 20 s of acquisition, CRISPR-targeted Ramos were activated with $2 \mu \mathrm{g} / \mathrm{ml}$ anti-human IgM F(ab') ${ }^{2}$; primary B

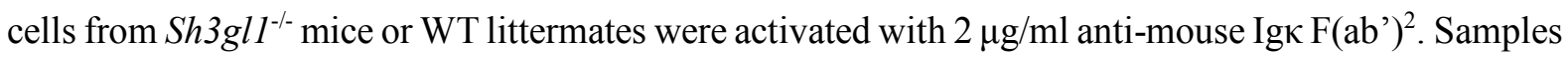
were acquired for 380 seconds, with addition of ionomycin $(5 \mu \mathrm{g} / \mathrm{ml})$ at $300 \mathrm{~s}$.

\section{Migration assay}

Directional migration assays were performed using 96-well Transwell plates with $5 \mu \mathrm{m}$ polycarbonate membranes (Sigma-Aldrich). Top compartments were coated with $0.5 \mu \mathrm{g} / \mathrm{ml} \mathrm{ICAM-1} \mathrm{or} 1 \mu \mathrm{g} / \mathrm{ml}$ antiIgא $\mathrm{F}(\mathrm{ab})_{2}$ in PBS for 1 hour, following by 20 min blocking in PBS-BSA (1\%). Bottom compartments contained RPMI with $1 \mu \mathrm{g} / \mathrm{ml} \mathrm{CXCL13,} 0.5 \mu \mathrm{g} / \mathrm{ml} \mathrm{CXCL12} \mathrm{or} 1 \mu \mathrm{g} / \mathrm{ml} \mathrm{CCL21} \mathrm{(all} \mathrm{from} \mathrm{R \& D}$ systems). Isolated chimera B cells, of known mCherry infection rates, were added to the top compartments and allowed to migrate at $37^{\circ} \mathrm{C}$ for 3 hours. mCherry percentages were quantified by flow cytometry.

\section{BioID2 proximity assay}

SH3GL1-BioID2-HA and empty-BioID2-HA constructs were cloned into a lentiviral expression vector (pLenti-Puro, Addgene) and used to create single-cell cloned stable Ramos cell lines. Expression was tested by western blot and correct localization confirmed by immunofluorescence using anti-BioID2 antibody (SS GD1, Novus Biologicals). $1 \times 10^{8}$ cells per sample were cultured in $1 \mu \mathrm{M}$ biotin in full RPMI for 14 hours. Cells were washed and lysed in RIPA buffer containing protease inhibitor cocktail. Protein concentration was determined using a BCA assay (Sigma) and biotinylated proteins were pulled down using a corresponding concentration of Dynabeads Streptavidin C1 (Thermo), as per 
manufacturer instructions. Biotinylated fraction was eluted in NuPAGE Sample Reducing Buffer (SDScontaining; Thermo), boiled at $95^{\circ} \mathrm{C}$ for $5 \mathrm{~min}$ and in-gel digestion performed. Peptide extracts were resolved on an EASY-Spray column (Thermo) using UltiMate 3000 RSLCnano System. Data acquisition was performed using an Orbitrap mass spectrometer (Thermo) controlled by Xcalibur software. Data analysis was performed using the MaxQuant bioinformatics suite, using LFQ (Label Free Quantification) algorithm.

\section{Seahorse assay}

Agilent Seahorse XF assay was performed as per manufacturer instructions. Briefly, B cells were seeded in poly-L-lysine-coated Agilent culture plate at 500 000/well for primary cells and 250000 for Ramos B cells. Cells were cultured in XF Base Medium supplemented with pyruvate, glutamine and glucose for 1 hour in a non-CO2 incubator at $37^{\circ} \mathrm{C}$. Stress compounds (all from Sigma) were diluted in assay medium and loaded at 10x concentration into the injection ports of the hydrated cartridge as follows: A- Oligomycin, $1 \mu \mathrm{M}$ final concentration; B- FCCP (Carbonyl cyanide-ptrifluoromethoxyphenyl hydrazone), $1.5 \mu \mathrm{M}$ final; C- Rotenone, $200 \mathrm{nM}$ final. XF Cell Mito Stress Test was run on a Seahorse XF 96 Analyzer with Wave software. Data was analyzed using Wave and GraphPad Prism.

\section{Immunization and serum antibody ELISA}

Sh $3 g l 1^{-/}$and WT littermates were immunized at 8-10 weeks of age by intraperitoneal injection of 50 $\mu \mathrm{g}$ NP-CGG in alum (Thermo Fisher). Blood samples were taken before immunization and at 7 and 14 days after immunization. Serum NP-specific antibodies were detected by ELISA on Nunc polysorb plates coated with $\mathrm{NP}_{7}$ or $\mathrm{NP}_{25}$ for capture. Total serum immunoglobulin levels were detected using SBA Clonotyping System HRP kit (Southern Biotech).

\section{ACKNOWLEDGEMENTS}

This work was supported by the European Research Council (Consolidator Grant 648228) and the Francis Crick Institute, which receives its core funding from Cancer Research UK (FC001185), the UK Medical Research Council (FC001185) and the Wellcome Trust (FC001185). We thank Emmanuel Boucrot and Jürgen Wienands for helpful discussions, Rebecca Newman for lentiGuide-mCherry plasmid and the Francis Crick Institute Science Technology Platforms for their help with mass spectrometry, cell sorting and next-generation sequencing.

\section{AUTHOR CONRIBUTIONS}


D.M. designed and performed the experiments and analyzed the data. L.W. performed live cell imaging.

N.E. provided knock out DG75 cells and advice. P.T. designed the experiments, analyzed data, and supervised the research. D.M. and P.T. prepared the manuscript.

\section{COMPETING INTERESTS STATEMENT}

All authors declare no competing interests.

\section{REFERENCES}

1. Lanzavecchia, A. Antigen-specific interaction between T and B cells. Nature 314, 537-539 (1985).

2. Amigorena, S., Drake, J. R., Webster, P. \& Mellman, I. Transient accumulation of new class II MHC molecules in a novel endocytic compartment in B lymphocytes. Nature 369, 113-120 (1994).

3. Hoogeboom, R. \& Tolar, P. Molecular Mechanisms of B Cell Antigen Gathering and Endocytosis. Curr. Top. Microbiol. Immunol. 393, 45-63 (2016).

4. Onabajo, O. O. et al. Actin-binding protein 1 regulates B cell receptor-mediated antigen processing and presentation in response to B cell receptor activation. J. Immunol. 180, 66856695 (2008).

5. Veselits, M. et al. Recruitment of Cbl-b to B cell antigen receptor couples antigen recognition to Toll-like receptor 9 activation in late endosomes. PLoS ONE 9, e89792 (2014).

6. Chatterjee, P., Tiwari, R. K., Rath, S., Bal, V. \& George, A. Modulation of antigen presentation and B cell receptor signaling in B cells of beige mice. J. Immunol. 188, 26952702 (2012).

7. Chaturvedi, A., Martz, R., Dorward, D., Waisberg, M. \& Pierce, S. K. Endocytosed BCRs sequentially regulate MAPK and Akt signaling pathways from intracellular compartments. Nat. Immunol. 12, 1119-1126 (2011).

8. Salisbury, J. L., Condeelis, J. S. \& Satir, P. Role of coated vesicles, microfilaments, and calmodulin in receptor-mediated endocytosis by cultured B lymphoblastoid cells. The Journal of Cell Biology 87, 132-141 (1980).

9. Brown, B. K. \& Song, W. The actin cytoskeleton is required for the trafficking of the B cell antigen receptor to the late endosomes. Traffic 2, 414-427 (2001).

10. Stoddart, A. et al. Lipid rafts unite signaling cascades with clathrin to regulate BCR internalization. Immunity 17, 451-462 (2002).

11. Busman-Sahay, K., Drake, L., Sitaram, A., Marks, M. \& Drake, J. R. Cis and trans regulatory mechanisms control AP2-mediated B cell receptor endocytosis via select tyrosine-based motifs. PLoS ONE 8, e54938 (2013).

12. Stoddart, A., Jackson, A. P. \& Brodsky, F. M. Plasticity of B cell receptor internalization upon conditional depletion of clathrin. Mol. Biol. Cell 16, 2339-2348 (2005).

13. Bonazzi, M. et al. Clathrin phosphorylation is required for actin recruitment at sites of bacterial adhesion and internalization. The Journal of Cell Biology 195, 525-536 (2011).

14. Kitaura, Y. et al. Control of the B cell-intrinsic tolerance programs by ubiquitin ligases $\mathrm{Cbl}$ and Cbl-b. Immunity 26, 567-578 (2007).

15. Satpathy, S. et al. Systems-wide analysis of BCR signalosomes and downstream phosphorylation and ubiquitylation. Mol. Syst. Biol. 11, 810-810 (2015).

16. Hou, P. et al. B cell antigen receptor signaling and internalization are mutually exclusive events. PLoS Biol. 4, e200 (2006).

17. Bai, J., Hu, Z., Dittman, J. S., Pym, E. C. G. \& Kaplan, J. M. Endophilin functions as a membrane-bending molecule and is delivered to endocytic zones by exocytosis. Cell 143, 430-441 (2010). 
18. Mim, C. et al. Structural basis of membrane bending by the N-BAR protein endophilin. Cell 149, 137-145 (2012).

19. Milosevic, I. et al. Recruitment of endophilin to clathrin-coated pit necks is required for efficient vesicle uncoating after fission. Neuron 72, 587-601 (2011).

20. Renard, H.-F. et al. Endophilin-A2 functions in membrane scission in clathrin-independent endocytosis. Nature 517, 493-496 (2015).

21. Boucrot, E. et al. Endophilin marks and controls a clathrin-independent endocytic pathway. Nature 517, 460-465 (2015).

22. Bertot, L. et al. Quantitative and Statistical Study of the Dynamics of Clathrin-Dependent and -Independent Endocytosis Reveal a Differential Role of EndophilinA2. Cell Rep 22, 15741588 (2018).

23. Watanabe, S. \& Boucrot, E. Fast and ultrafast endocytosis. Curr. Opin. Cell Biol. 47, 64-71 (2017).

24. Sánchez-Barrena, M. J. et al. Bin2 is a membrane sculpting N-BAR protein that influences leucocyte podosomes, motility and phagocytosis. PLoS ONE 7, e52401 (2012).

25. Soukup, S.-F. et al. A LRRK2-Dependent EndophilinA Phosphoswitch Is Critical for Macroautophagy at Presynaptic Terminals. Neuron 92, 829-844 (2016).

26. Genet, G. et al. Endophilin-A2 dependent VEGFR2 endocytosis promotes sprouting angiogenesis. Nat Comms 10, 2350-15 (2019).

27. Murdoch, J. D. et al. Endophilin-A Deficiency Induces the Foxo3a-Fbxo32 Network in the Brain and Causes Dysregulation of Autophagy and the Ubiquitin-Proteasome System. Cell Rep 17, 1071-1086 (2016).

28. Nguyen, M., Wong, Y. C., Ysselstein, D., Severino, A. \& Krainc, D. Synaptic, Mitochondrial, and Lysosomal Dysfunction in Parkinson's Disease. Trends Neurosci. 42, 140-149 (2019).

29. Doench, J. G. et al. Optimized sgRNA design to maximize activity and minimize off-target effects of CRISPR-Cas9. Nat. Biotechnol. 34, 184-191 (2016).

30. Wang, T. et al. Identification and characterization of essential genes in the human genome. Science (New York, N.Y.) aac7041 (2015). doi:10.1126/science.aac7041

31. Kim, D. I. et al. An improved smaller biotin ligase for BioID proximity labeling. Mol. Biol. Cell 27, 1188-1196 (2016).

32. Burbage, M. et al. Tuning of in vivo cognate B-T cell interactions by Intersectin 2 is required for effective anti-viral B cell immunity. Elife 7, 113 (2018).

33. Mercier, V. et al. ALG-2 interacting protein-X (Alix) is essential for clathrin-independent endocytosis and signaling. Sci Rep 6, 26986-15 (2016).

34. Vanshylla, K. et al. Grb2 and GRAP connect the B cell antigen receptor to Erk MAP kinase activation in human B cells. Sci Rep 8, 4244-17 (2018).

35. Phan, T. G. et al. B cell receptor-independent stimuli trigger immunoglobulin (Ig) class switch recombination and production of IgG autoantibodies by anergic self-reactive B cells. J. Exp. Med. 197, 845-860 (2003).

36. Brink, R. et al. The SW(HEL) system for high-resolution analysis of in vivo antigen-specific T-dependent B cell responses. Methods Mol. Biol. 1291, 103-123 (2015).

37. Phelan, J. D. et al. A multiprotein supercomplex controlling oncogenic signalling in lymphoma. Nature 560, 387-391 (2018).

38. Wang, T. et al. Gene Essentiality Profiling Reveals Gene Networks and Synthetic Lethal Interactions with Oncogenic Ras. Cell 168, 890-903.e15 (2017).

39. Seligman, P. A., Kovar, J. \& Gelfand, E. W. Lymphocyte proliferation is controlled by both iron availability and regulation of iron uptake pathways. Pathobiology 60, 19-26 (1992).

40. Cronin, S. J. F., Woolf, C. J., Weiss, G. \& Penninger, J. M. The Role of Iron Regulation in Immunometabolism and Immune-Related Disease. Front Mol Biosci 6, 116 (2019).

41. Ferguson, S. M. et al. Coordinated actions of actin and BAR proteins upstream of dynamin at endocytic clathrin-coated pits. Dev. Cell 17, 811-822 (2009).

42. Sundborger, A. et al. An endophilin-dynamin complex promotes budding of clathrin-coated vesicles during synaptic vesicle recycling. J. Cell. Sci. 124, 133-143 (2011).

43. Wong, K. A. et al. Intersectin (ITSN) family of scaffolds function as molecular hubs in protein interaction networks. PLoS ONE 7, e36023 (2012). 
44. Burbage, M. et al. Tuning of in vivo cognate B-T cell interactions by Intersectin 2 is required for effective anti-viral B cell immunity. Elife 7, 113 (2018).

45. Pillai, S. \& Cariappa, A. The follicular versus marginal zone B lymphocyte cell fate decision. Nat. Rev. Immunol. 9, 767-777 (2009).

46. Pozzi, B. et al. The endocytic adaptor Eps15 controls marginal zone B cell numbers. PLoS ONE 7, e50818 (2012).

47. Kurz, T., Eaton, J. W. \& Brunk, U. T. The role of lysosomes in iron metabolism and recycling. Int. J. Biochem. Cell Biol. 43, 1686-1697 (2011).

48. Yambire, K. F. et al. Impaired lysosomal acidification triggers iron deficiency and inflammation in vivo. Elife 8, 807 (2019).

49. Weber, R. A. et al. Maintaining Iron Homeostasis Is the Key Role of Lysosomal Acidity for Cell Proliferation. Mol. Cell 77, 645-655.e7 (2020).

50. Kwak, K. et al. Intrinsic properties of human germinal center B cells set antigen affinity thresholds. Sci Immunol 3, eaau6598 (2018).

51. Shalem, O. et al. Genome-scale CRISPR-Cas9 knockout screening in human cells. Science (New York, N.Y.) 343, 84-87 (2014).

52. Li, W. et al. MAGeCK enables robust identification of essential genes from genome-scale CRISPR/Cas9 knockout screens. Genome Biol. 15, 554 (2014).

53. Nowosad, C. R. \& Tolar, P. Plasma Membrane Sheets for Studies of B Cell Antigen Internalization from Immune Synapses. Methods Mol. Biol. 1584, 77-88 (2017).

54. Nowosad, C. R., Spillane, K. M. \& Tolar, P. Germinal center B cells recognize antigen through a specialized immune synapse architecture. Nat. Immunol. 17, 870-877 (2016).

55. Roper, S. I. et al. B cells extract antigens at Arp2/3-generated actin foci interspersed with linear filaments. Elife 8, 489 (2019).

\section{TABLES}

Supplemental Table 1. Internalization analysis results from GeCKO genome-wide CRISPR library.

Supplemental Table 2. Internalization analysis results from Brunello genome-wide CRISPR library.

Supplemental Table 3. Internalization analysis results from validation custom CRISPR library.

Supplemental Table 4. Endophilin A2 interaction partners detected by BioID2.

Supplemental Table 5. RNAseq analysis of sorted transitional, follicular naïve and CD40L cultured B cells from WT and Sh $3 g l 1^{-/-}$littermates. Raw and TPM (transcript per million) values are provided for 3 mice per genotype.

\section{VIDEOS}

Video 1. Live TIRF imaging of a Ramos cell expressing endophilin A2-GFP (green) and mCherryClathrin LC (magenta). The cell is interacting with anti-IgM F(ab') $)_{2}$-loaded PMS (blue). Simple ratio bleach correction (Fiji) was performed on the antigen channel. Yellow boxes indicate instances of endophilin A2-GFP recruitment to antigen clusters in the absence of clathrin LC signal. Scale bar $=5$ $\mu \mathrm{m}$. 